\title{
Fluctuation of the primitive of Hardy's function
}

\author{
Matti Jutila
}

To the memory of S. Srinivasan

\begin{abstract}
We consider oscillatory properties of the primitive of Hardy's function including a certain Gibbs phenomenon due to M. A. Korolev.
\end{abstract}

Keywords. Riemann's zeta-function, Hardy's function, the Gibbs phenomenon.

2010 Mathematics Subject Classification. 11M06.

\section{Introduction}

Hardy's function,

$$
Z(t)=\chi\left(\frac{1}{2}+i t\right)^{-1 / 2} \zeta\left(\frac{1}{2}+i t\right),
$$

a real function for real $t$, determines the behavior of the zeta-function on the critical line. Here $\chi(s)=2^{s} \pi^{s-1} \sin \left(\frac{1}{2} \pi s\right) \Gamma(1-s)$ is as in the functional equation $\zeta(s)=\chi(s) \zeta(1-s)$ for Riemann's zeta-function. We refer to the monograph [Iv13] of A. Ivić for an account of the theory of Hardy's function.

The primitive

$$
F(T)=\int_{0}^{T} Z(t) \mathrm{d} t
$$

of Hardy's function measures the balance between its positive and negative values. The sharpest (and best possible) estimate for $F(T)$ is $O\left(T^{1 / 4}\right.$ ) due to M. A. Korolev [Kor08] (we gave another proof in [Jut09]), and this means that the balance is globally quite nice and well understood. The local behavior is a different matter and its irregularity is the main topic of this paper.

We study the local behavior of $Z(t)$ in terms of the integrals

$$
I\left(T_{1}, T_{2}\right)=\int_{T_{1}}^{T_{2}} Z(t) \mathrm{d} t=F\left(T_{2}\right)-F\left(T_{1}\right)
$$

For orientation, let us consider the mean value

$$
\frac{1}{T} \int_{T}^{2 T} I(t, t+U)^{2} \mathrm{~d} t
$$

We showed in [Jut15] that this is $\asymp U$ for $1 / \log T \ll U \leq \sqrt{T}$. Thus, at first sight, the expectation of $|I(t, t+U)|$ seems to be about $U^{1 / 2}$, but this heuristics is incorrect as we pointed out in [Jut11]. Namely, the graph of $F(t)$ has occasionally jumps or drops giving abnormally big contributions to certain integrals $I(t, t+U)$, and we are going to specify such cases. $1)$ :

Our analysis of the function $F(T)$ is based on a formula of Atkinson type (see [Jut09], Theorem

$$
F(T)=S_{1}(T, L)+S_{2}\left(T, L^{\prime}\right)+O\left((\log T)^{5 / 4}\right),
$$

where $S_{1}(T, L)$ and $S_{2}\left(T, L^{\prime}\right)$ are certain sums of length $L$ and $L^{\prime}$, respectively, with $L, L^{\prime} \asymp \sqrt{T}$. An application for this is a formula for $F(T)$ (Theorem 2 in [Jut09]) in terms of the step function

$$
K(x)= \begin{cases}0 & \text { for } 0 \leq x<1 / 4 \text { and } 3 / 4<x \leq 1 \\ 2 \pi & \text { for } 1 / 4<x<3 / 4\end{cases}
$$

We thank episciences.org for providing open access hosting of the electronic journal Hardy-Ramanujan Journal 
Write $\sqrt{T / 2 \pi}=P+\vartheta$ with $P \in \mathbb{N}$ and $0 \leq \vartheta<1$. Let $\vartheta_{0}=\min (|\vartheta-1 / 4|,|\vartheta-3 / 4|)$. Then for $\vartheta_{0} \neq 0$ we have

$$
F(T)=(T / 2 \pi)^{1 / 4}(-1)^{P} K(\vartheta)+O\left(T^{1 / 6} \log T\right)+O\left(\min \left(T^{1 / 4}, T^{1 / 8} \vartheta_{0}^{-3 / 4}\right)\right) .
$$

Further, for $\vartheta_{0}=0$, that is for $\vartheta=j / 4$ with $j=1$ or 3 , we have

$$
F(T)=(T / 2 \pi)^{1 / 4}(-1)^{P}\left(\pi+(-1)^{(j-1) / 2}(\pi / 3)\right)+O\left(T^{1 / 6} \log T\right) .
$$

We are going to keep $P$ fixed writing $t(\vartheta)=2 \pi(P+\vartheta)^{2}$. Thus $\sqrt{t(\vartheta) / 2 \pi}=P+\vartheta$. Further, fixing henceforth the meaning of $T$, we let it stand for the number $T=t(0)=2 \pi P^{2}$ which will serve as an approximation for $t(\vartheta)$. Our main topic will be an analysis of $F(t(\vartheta))$ as a function of $\vartheta$.

The formula (1.2) shows that $F(t)$ is expected to change rapidly when $t$ passes $t(1 / 4)$ or $t(3 / 4)$. In fact, M. A. Korolev has shown for $F(t)$ a property analogous to the Gibbs phenomenon familiar from the theory of the Fourier series. We are going to return to this topic in sec. 5. Therefore the interesting cases will be

$$
\vartheta=\frac{j}{4}+\delta \text { with } j=1 \text { or } 3 \text { and }|\delta| \leq 1 / 4,
$$

especially when $\delta$ is relatively small. An error term like the second one in (1.2) is apparently inevitable since the function $K$ is discontinuous while $F$ is continuous. We are going to to consider this error term more closely in sec. 6 . In [Jut11] we removed this error term on replacing $K(\vartheta)$ by a smoothed version $\tilde{K}(\vartheta)$. Its definition involved the Airy function which will appear in this paper as well.

Consider the jump of $F(t)$ near $t(j / 4)$ using (1.2). For $P$ even and $j=1$, say, and writing $t_{1}=t\left(1 / 4-c_{1} T^{-1 / 6}\right), t_{2}=t\left(1 / 4+c_{1} T^{-1 / 6}\right)$, we have

$$
c_{2} T^{1 / 4} \leq I\left(t_{1}, t_{2}\right) \leq c_{3} T^{1 / 4}
$$

where the $c_{i}$ are suitable constants; choose $c_{1}$ sufficiently large. Note that $t_{2}-t_{1} \asymp T^{1 / 3}$, so the bound (1.4) exceeds the "expected" order $\sqrt{t_{2}-t_{1}} \asymp T^{1 / 6}$ by a factor about $T^{1 / 12}$. The mean bias of $Z(t)$ in the positive direction in the interval $\left[t_{1}, t_{2}\right]$ is

$$
\frac{I\left(t_{1}, t_{2}\right)}{t_{2}-t_{1}} \asymp T^{-1 / 12} \text {. }
$$

Naturally one would expect the same property to hold even for suitable subintervals of this intervals as well, and this will be shown in Theorem 2. However, this conclusion cannot be drawn directly from (1.2) which gives poor information in the critical ranges. Therefore we adopt another approach to $F(t)$ using an approximate functional equation proved in [Jut17].

This approximate functional equation is a generalization of the formula (1.1), where the parameters $L$ and $L^{\prime}$ are now allowed to vary fairly freely, depending however on each other. In particular, for $t(0) \leq t<t(1)$, that is for $T \leq t \leq T+2 \sqrt{2 \pi T}+2 \pi$, we have (see [Jut17], Theorem 3 )

$$
F(t)=S_{1}\left(t, L_{0}\right)+O\left(T^{c}\right),
$$

where $L_{0} \asymp T^{1 / 4}$ and $c<1 / 6$; the value $c=27 / 164=1 / 6-1 / 492$ is applicable.

\section{A formula for $F(t)$}

The terms in the sum $S_{1}\left(t, L_{0}\right)$ in (1.5) are somewhat complicated, but the following simplified version (see [Jut09], Sec. 4.1) suffices for our purposes: for $t=t(\vartheta)$, we have

$$
\begin{aligned}
S_{1}\left(t, L_{0}\right)= & 2 \sqrt{2}(t / 2 \pi)^{1 / 4}(-1)^{P} \sum_{0 \leq n \leq L_{0}}\left(1+O\left(\left(n+\frac{1}{2}\right)^{2} / T\right)\right)\left(n+\frac{1}{2}\right)^{-1}(-1)^{n(n+1) / 2} \\
& \times \sin \left(2 \pi\left(n+\frac{1}{2}\right) \vartheta+A(t)\left(n+\frac{1}{2}\right)^{3}+O\left(\left(n+\frac{1}{2}\right)^{5} T^{-3 / 2}\right)\right)
\end{aligned}
$$


where $A(t)=\frac{1}{12} \sqrt{2 \pi^{3}} t^{-1 / 2}$. As further simplifications, we omit the error terms here, replacing moreover $t$ by $T$ in $A(t)$ and in the factor $(t / 2 \pi)^{1 / 4}$. Then, writing $A(T)=A$, we have

$$
S_{1}\left(t(\vartheta), L_{0}\right)=2 \sqrt{2}(T / 2 \pi)^{1 / 4}(-1)^{P} S(\vartheta)+O(1),
$$

where the dependence on $\vartheta$ is contained in the sum

$$
S(\vartheta)=\sum_{0 \leq n \leq L_{0}}\left(n+\frac{1}{2}\right)^{-1}(-1)^{n(n+1) / 2} \sin \left(2 \pi\left(n+\frac{1}{2}\right) \vartheta+A\left(n+\frac{1}{2}\right)^{3}\right) .
$$

Hence (1.5) and (2.1) give the formula

$$
F(t)=F(t(\vartheta))=2 \sqrt{2}(T / 2 \pi)^{1 / 4}(-1)^{P} S(\vartheta)+O\left(T^{c}\right)
$$

which will be the basis for our subsequent analysis.

Now the change of $F(t)$ in terms of $\vartheta$ is reduced to the change of $S(\vartheta)$ : if $t_{1}=t\left(\vartheta_{1}\right)$ and $t_{2}=t\left(\vartheta_{2}\right)$, then

$$
F\left(t_{2}\right)-F\left(t_{1}\right)=2 \sqrt{2}(T / 2 \pi)^{1 / 4}(-1)^{P} \int_{\vartheta_{1}}^{\vartheta_{2}} S^{\prime}(\vartheta) \mathrm{d} \vartheta+O\left(T^{c}\right) .
$$

Thus it remains to evaluate the derivative

$$
S^{\prime}(\vartheta)=2 \pi \sum_{0 \leq n \leq L_{0}}(-1)^{n(n+1) / 2} \cos \left(2 \pi\left(n+\frac{1}{2}\right) \vartheta+A\left(n+\frac{1}{2}\right)^{3}\right),
$$

the interesting cases being $\vartheta=j / 4+\delta$ with $j=1$ or 3 . The main point is to show that $S^{\prime}(\vartheta)$ is of constant sign in suitable $\delta$ - intervals. Then $S(\vartheta)$ will be monotonic in this interval, and by (2.4) we may estimate changes of the function $F(t)$.

\section{A formula for $S^{\prime}(\vartheta)$}

The cosine factor in $(2.5)$ with $\vartheta=\frac{j}{4}+\delta$ is

$$
\cos \left(2 \pi\left(n+\frac{1}{2}\right) \frac{j}{4}\right) \cos \left(2 \pi\left(n+\frac{1}{2}\right) \delta+A\left(n+\frac{1}{2}\right)^{3}\right)-\sin \left(2 \pi\left(n+\frac{1}{2}\right) \frac{j}{4}\right) \sin \left(2 \pi\left(n+\frac{1}{2}\right) \delta+A\left(n+\frac{1}{2}\right)^{3}\right) .
$$

The factors

$$
\begin{gathered}
(-1)^{n(n+1) / 2} \cos \left(2 \pi\left(n+\frac{1}{2}\right) \frac{j}{4}\right), \\
(-1)^{n(n+1) / 2} \sin \left(2 \pi\left(n+\frac{1}{2}\right) \frac{j}{4}\right)
\end{gathered}
$$

depend on $n$ modulo 4 . Let $n$ run over the residues $0,1,2,3(\bmod 4)$. Then the numbers $(3.1)$ for $j=1$ or 3 give the vector

$$
\frac{1}{\sqrt{2}}(-1)^{(j-1) / 2}(1,1,1,1)
$$

whereas the numbers (3.2) give in both cases the vector

$$
\frac{1}{\sqrt{2}}(1,-1,1,-1)
$$

Hence the formula (2.5) becomes

$$
\begin{array}{r}
S^{\prime}\left(\frac{j}{4}+\delta\right)=\sqrt{2} \pi(-1)^{(j-1) / 2} \sum_{0 \leq n \leq L_{0}} \cos \left(2 \pi\left(n+\frac{1}{2}\right) \delta+A\left(n+\frac{1}{2}\right)^{3}\right) \\
-\sqrt{2} \pi \sum_{0 \leq n \leq L_{0}}(-1)^{n} \sin \left(2 \pi\left(n+\frac{1}{2}\right) \delta+A\left(n+\frac{1}{2}\right)^{3}\right) .
\end{array}
$$


Next we turn the sums here into integrals using van der Corput's lemma (see [Ti86], Lemma 4.8). For this purpose, we suppose that

$$
|\delta| \leq a, \quad L_{0}=b T^{1 / 4}
$$

where $a$ and $b$ are sufficiently small positive constants. Then for the function

$$
f(x)=\left(x+\frac{1}{2}\right) \delta+(A / 2 \pi)\left(x+\frac{1}{2}\right)^{3}
$$

we have $\left|f^{\prime}(x)\right| \leq \theta$ for $0 \leq x \leq L_{0}$ with $0<\theta<1$ if $a$ and $b$ are suitably chosen; recall that $A \asymp T^{-1 / 2}$. Then van der Corput's lemma gives the following formula for the sum in (3.3):

$$
\sum_{0 \leq n \leq L_{0}} \cos \left(2 \pi\left(n+\frac{1}{2}\right) \delta+A\left(n+\frac{1}{2}\right)^{3}\right)=\Re \int_{0}^{L_{0}} e(f(x)) \mathrm{d} x+O(1) .
$$

Also, by the same lemma, we have

$$
\sum_{0 \leq n \leq L_{0}}(-1)^{n} \sin \left(2 \pi\left(n+\frac{1}{2}\right) \delta+A\left(n+\frac{1}{2}\right)^{3}\right)=O(1) .
$$

To verify this, consider the sums over even and odd $n$ separately and note that the difference of the respective integrals is bounded.

We extend the integration in (3.6) to infinity. This is allowed, by integration by parts, if $\left|f^{\prime}(x)\right| \gg 1$ for $x \geq L_{0}$. This holds if $a$ and $b$ in (3.5) are chosen in such a way that $a$ is small in comparison with $b^{2}$.

By (3.3), (3.4), (3.6), (3.7), and the last made observation, we obtain

$$
S^{\prime}\left(\frac{j}{4}+\delta\right)=\sqrt{2} \pi(-1)^{(j-1) / 2} B(\delta)+O(1)
$$

where

$$
B(u)=\int_{0}^{\infty} \cos \left(A x^{3}+2 \pi u x\right) \mathrm{d} x
$$

is an Airy integral. This has occurred in [Jut11] and also in the Bombieri-Iwaniec method [BoIw86]. It can be expressed in terms of Bessel functions as follows (see [Wat66], p. 190) :

$$
\begin{gathered}
B(u)=\frac{\sqrt{2 \pi u}}{3 \sqrt{A}} K_{1 / 3}\left(\frac{2(2 \pi u)^{3 / 2}}{3 \sqrt{3 A}}\right) \text { for } u>0, \\
B(u)=\frac{\pi \sqrt{2 \pi|u|}}{3 \sqrt{3 A}}\left(J_{1 / 3}\left(\frac{2(2 \pi|u|)^{3 / 2}}{3 \sqrt{3 A}}\right)+J_{-1 / 3}\left(\frac{2(2 \pi|u|)^{3 / 2}}{3 \sqrt{3 A}}\right)\right) \text { for } u<0 .
\end{gathered}
$$

Letting $u$ tend to zero, we get

$$
B(0)=\sqrt{2 \pi} 3^{-2 / 3} \Gamma(2 / 3)^{-1} T^{1 / 6} .
$$

Avoiding Bessel functions, this can be also verified directly using the theory of the gamma-function. In terms of the Airy function

$$
\operatorname{Ai}(z)=\int_{0}^{\infty} \cos \left(\frac{x^{3}}{3}+x z\right) \mathrm{d} x
$$

we have

$$
B(u)=\sqrt{2 / \pi} \operatorname{Ai}\left(2 \sqrt{2 \pi} T^{1 / 6} u\right) T^{1 / 6} .
$$

With the Gibbs Phenomenon in mind, we note the formula (see [Jut11], eq. (2.13))

$$
\int_{-\infty}^{0} B(u) \mathrm{d} u=\frac{1}{3}=\frac{1}{2 \pi} \int_{-\infty}^{0} \operatorname{Ai}(v) \mathrm{d} v .
$$


Let $B\left(u_{0}\right)=0$ with $u_{0}<0$ and $\left|u_{0}\right|$ minimal, and let $v_{0}$ be the corresponding zero for $\operatorname{Ai}(v)$, that is

$$
v_{0}=2 \sqrt{2 \pi} T^{1 / 6} u_{0}
$$

by (3.12). Since $v_{0}$ is a numerical constant, we have $\left|u_{0}\right| \asymp T^{-1 / 6}$. A crucial fact for the occurrence of the Gibbs phenomenon will be that

$$
\int_{u_{0}}^{0} B(u) \mathrm{d} u=\frac{1}{3}+c_{0}=\frac{1}{2 \pi} \int_{v_{0}}^{0} \operatorname{Ai}(v) \mathrm{d} v
$$

where $c_{0}$ is positive constant. This can be verified by numerical calculations in terms of Airy's function. Alternatively, we have

$$
\int_{u_{0}}^{0} B(u) \mathrm{d} u=\frac{1}{3}-\int_{-\infty}^{u_{0}} B(u) \mathrm{d} u=\frac{1}{3}-\frac{1}{2 \pi} \int_{-\infty}^{v_{0}} \operatorname{Ai}(v) \mathrm{d} v
$$

where the last mentioned integral is negative; this property is natural in view of the oscillatory behavior of Airy's function and the definition of $v_{0}$.

\section{Variation of the function $F(t)$}

We combine now the formulae (2.4) and (3.8) giving immediately the following theorem.

Theorem 1. Define $t_{i, j}=t\left(j / 4+\delta_{i}\right)$ for $i=1,2$ and $j=1,3$. Suppose that $\left|\delta_{i}\right| \leq a$, where $a$ is a sufficiently small constant. Then

$$
F\left(t_{2, j}\right)-F\left(t_{1, j}\right)=4 \pi(T / 2 \pi)^{1 / 4}(-1)^{P}(-1)^{(j-1) / 2} \int_{\delta_{1}}^{\delta_{2}} B(u) \mathrm{d} u+O\left(T^{c}\right)+O\left(\left|\delta_{2}-\delta_{1}\right| T^{1 / 4}\right) .
$$

As an application of (4.1) we generalize the estimate for $I\left(t_{1}, t_{2}\right)$ in (1.4).

Theorem 2. Let $t_{1}<t_{2}$ with the $t_{i}$ lying in the interval

$$
\left[t(j / 4)-d T^{1 / 3}, t(j / 4)+d T^{1 / 3}\right],
$$

where $j=1$ or 3 and $d$ is a sufficiently small constant. Suppose that $U=t_{2}-t_{1} \gg c_{4} T^{c+1 / 12}$, where $c_{4}$ is a sufficiently large constant. Then

$$
c_{5} U T^{-1 / 12} \leq(-1)^{P}(-1)^{(j-1) / 2} I\left(t_{1}, t_{2}\right) \leq c_{6} U T^{-1 / 12},
$$

where $c_{5}$ and $c_{6}$ are suitable positive constants.

Proof. Interpret the numbers $t_{i}$ in terms of the parameters $\delta_{i}$ as in Theorem 1 . Then by our assumption on $t_{i}$ we see that $\left|\delta_{i}\right| \leq d_{1} T^{-1 / 6}$, where $d_{1}$ is a small constant depending on $d$. Also, $\delta_{2}-\delta_{1} \asymp T^{-1 / 2} U$. Hence the arguments of the Bessel functions involved in the formulae for $B(u)$ with $u \in\left[\delta_{1}, \delta_{2}\right]$ are below a small constant depending on $d$. Therefore we may use the following well-known formulae (see e. g. [Leb72], sec. 5.16): for $0<x \ll 1$, we have

$$
\begin{aligned}
J_{ \pm 1 / 3}(x) & =\frac{x^{ \pm 1 / 3}}{2^{ \pm 1 / 3} \Gamma(1 \pm 1 / 3)}(1+O(x)), \\
K_{1 / 3}(x) & =2^{-2 / 3} \Gamma(1 / 3) x^{-1 / 3}(1+O(x)) .
\end{aligned}
$$

Thus we see that $B(u) \asymp T^{1 / 6}$; it is essential that this value is positive. Hence the leading term in (4.1) is $\asymp T^{-1 / 12} U$. This exceeds the error terms if the constant $c_{4}$ is taken sufficiently large. This completes the proof of the theorem. 


\section{The Gibbs Phenomenon for $F(t)$}

To exhibit this phenomenon, consider the value $F\left(t\left(j / 4+u_{0}\right)\right)$, where $u_{0}$ is as in sec. 3 , thus $B\left(u_{0}\right)=0$, $u_{0}<0,\left|u_{0}\right| \asymp T^{-1 / 6}$, and $\left|u_{0}\right|$ is minimal. Write

$$
F\left(t\left(j / 4+u_{0}\right)\right)=F(t(j / 4))-\left(F(t(j / 4))-F\left(t\left(j / 4+u_{0}\right)\right)\right) .
$$

The first term on the right is given by (1.3), and the second term is expressed by (4.1) with $\delta_{1}=u_{0}$ and $\delta_{2}=0$. Using also (3.13), we obtain

$$
F\left(t\left(j / 4+u_{0}\right)\right)=\pi(T / 2 \pi)^{1 / 4}(-1)^{P}\left(1-(-1)^{(j-1) / 2}\left(1+4 c_{0}\right)\right)+O\left(T^{1 / 6} \log T\right) .
$$

In particular, for $P$ even and $j=3$, we have

$$
F\left(t\left(3 / 4+u_{0}\right)\right)=2 \pi(T / 2 \pi)^{1 / 4}\left(1+2 c_{0}\right)+O\left(T^{1 / 6} \log T\right) .
$$

This should be compared with (1.2).

Numerically, it was shown by M. A. Korolev that there are large values of T such that

$$
F(T)=2 \pi(T / 2 \pi)^{1 / 4}(1+0.277435 \ldots+o(1)) .
$$

\section{Remarks on the formula (1.2)}

Consider the formula (1.2) for $F(t)$ with $t=t(\vartheta)=t(j / 4+\delta)$, where $j=1$ or 3 and $0<|\delta|<1 / 4$. For simplicity we replace $t$ by $T=t(0)$ in the leading term; this can be done with a negligible error $O\left(T^{-1 / 4}\right)$. We write a slightly modified version of (1.2) as follows:

$$
F(t)=(T / 2 \pi)^{1 / 4}(-1)^{P} K(\vartheta)+G(t),
$$

where

$$
G(t) \ll T^{1 / 6} \log T+\min \left(T^{1 / 4}, T^{1 / 8} \vartheta_{0}^{-3 / 4}\right)
$$

with $\vartheta_{0}=|\delta|$. One may ask whether or not this represents the true order of $G(t)$. It will turn out that the answer depends on the sign of $\delta$. Therefore we are going to consider the cases $\delta>0$ and $\delta<0$ separately. In any case, the "trivial" estimate $G(t) \ll T^{1 / 4}$ gives the true order if $\vartheta_{0} \ll T^{-1 / 6}$ as we observed in sec. 4 . Also, the case $\vartheta_{0} \gg T^{-1 / 18}$ is less interesting because then the dependence on $\vartheta_{0}$ disappears in (6.2). Therefore we assume henceforth that

$$
c_{7} T^{-1 / 6} \leq \vartheta_{0} \ll T^{-1 / 18-\varepsilon},
$$

where $c_{7}$ is a sufficiently large constant and $\varepsilon$ is a small positive constant.

Though our present interest is the function $F(t)$ itself, it will be helpful to start from its differences such as those in (4.1), where the $\delta_{i}$ are of the same sign with $\left|\delta_{i}\right|$ lying in the interval (6.3).

The Airy function $B(u)$ in (4.1) is expressed in terms of Bessel functions by the formulae (3.10) and (3.11), and then we have values of $J_{ \pm 1 / 3}(x)$ and $K_{1 / 3}(x)$ for $x \gg 1$ to deal with. These functions behave quite differently in this range: the $J$ - Bessel functions are sign-changing and oscillatory, whereas the $K$ - Bessel function is positive and of exponential decay, more precisely (see [Leb72], eq. $(5.11 .9))$

$$
K_{1 / 3}(x)=\sqrt{\frac{\pi}{2 x}} e^{-x}\left(1+O\left(x^{-1}\right)\right) .
$$

Consequently, the behavior of $F(t)$ is curiously asymmetrical near $t(1 / 4)$ and $t(3 / 4)$. 
The case $\delta>0$. Applied to (3.10), the formula (6.4) yields the following formula: for $u>0$ and $u \gg T^{-1 / 6}$, we have

$$
B(u)=2^{-5 / 4} 3^{-1 / 4} \pi^{1 / 4} A^{-1 / 4} u^{-1 / 4} \exp \left(-\frac{2(2 \pi u)^{3 / 2}}{3 \sqrt{3 A}}\right)\left(1+O\left(T^{-1 / 4} u^{-3 / 2}\right)\right) .
$$

Let now $t=t(\vartheta)=t(j / 4+\delta)$ and $t^{\prime}=t\left(j / 4+\delta^{\prime}\right)$ with $\delta^{\prime}=T^{-1 / 14}$. This value is determined from the equation

$$
T^{1 / 4} \delta^{\prime}=T^{1 / 8}\left(\delta^{\prime}\right)^{-3 / 4}=T^{5 / 28}
$$

this condition will be motivated in a moment. Suppose that $\delta=\vartheta_{0}$ lies in the interval

$$
c_{7} T^{-1 / 6} \leq \delta \ll \delta^{\prime}
$$

which is a subinterval of (6.3). We write

$$
F(t)=\left(F(t)-F\left(t^{\prime}\right)\right)+F\left(t^{\prime}\right),
$$

where the terms will be treated differently. First, by (6.1) and (6.2), we have

$$
F\left(t^{\prime}\right)=(T / 2 \pi)^{1 / 4}(-1)^{P} K(\vartheta)+O\left(T^{5 / 28}\right) .
$$

Further,

$$
F\left(t^{\prime}\right)-F(t)=4 \pi(T / 2 \pi)^{1 / 4}(-1)^{P}(-1)^{(j-1) / 2} \int_{\vartheta_{0}}^{\delta^{\prime}} B(u) \mathrm{d} u+O\left(T^{5 / 28}\right)
$$

by (4.1). The parameter $\delta^{\prime}$ was chosen so as to balance the two error terms above. All in all, using here the formula (6.5) and integration by parts, we obtain

$$
F(t)=(T / 2 \pi)^{1 / 4}(-1)^{P} K(\vartheta)+O(E),
$$

where

$$
E \ll T^{5 / 28}+T^{1 / 8} \vartheta_{0}^{-3 / 4} \exp \left(-\frac{2\left(2 \pi \vartheta_{0}\right)^{3 / 2}}{3 \sqrt{3 A}}\right) .
$$

This sharpens (6.2) in the range (6.6). Recall that $\vartheta=j / 4+\vartheta_{0}$ for $j=1$ or 3 .

The case $\delta<0$. It will turn out that the estimate (6.2) is best possible for $\vartheta_{0}$ lying in the range (6.3). More precisely, letting $D$ be a parameter lying in this range, we show that there is number $\vartheta_{0} \asymp D$ such that

$$
G\left(t\left(j / 4-\vartheta_{0}\right)\right) \asymp T^{1 / 8} D^{-3 / 4} .
$$

For a proof, we study differences of $F(t)$ such as those in (4.1), with $\delta_{1}, \delta_{2}<0$ and $\left|\delta_{i}\right| \asymp D$. The Airy function $B(u)$ for $u<0$ is expressed in terms of $J$ - Bessel functions according to (3.11). We might apply the well-known asymptotic formula for $J$ - Bessel functions. However, we prefer to appeal to an asymptotic formula for Airy's function due to E. Bombieri and H. Iwaniec (see [BoIw86], Lemma 2.5):

$$
\int_{0}^{\infty} \cos \left(2 \pi x^{3}-6 \pi y x\right) \mathrm{d} x=(6 \sqrt{y})^{-1 / 2} \cos \left(-4 \pi y^{3 / 2}+\pi / 4\right)+O\left(y^{-1}\right) \text { for } y \geq 1 .
$$

We apply this to $B(u)$ for $u<0$ making a change of the variable in (3.9). Supposing that $|u| \asymp D$ we have

$$
B(u)=2^{-1 / 4} 3^{-1 / 4} \pi^{1 / 4} A^{-1 / 4}|u|^{-1 / 4} \cos \left(-\frac{2(2 \pi|u|)^{3 / 2}}{3 \sqrt{3 A}}+\pi / 4\right)+O\left(D^{-1}\right) .
$$


We show next that $\left|F\left(t_{2, j}\right)-F\left(t_{1, j}\right)\right|$ in (4.1) can be made "large" with with $\left|\delta_{i}\right| \asymp D$ suitably chosen. Considering a wave of the cosine in (6.8), we choose the $\delta_{i}$ in such a way that $\left|\delta_{2}-\delta_{1}\right| \asymp$ $D^{-1 / 2} T^{-1 / 4}$ and $|B(u)| \asymp D^{-1 / 4} T^{1 / 8}$ for $\delta_{1} \leq u \leq \delta_{2}$. Then by Theorem 1 we have

$$
\left|F\left(t_{2, j}\right)-F\left(t_{1, j}\right)\right| \asymp T^{1 / 8} D^{-3 / 4} ;
$$

note that the right hand side dominates the error terms in (4.1). This proves (6.7). Namely, either for $F\left(t_{1, j}\right)$ or for $F\left(t_{2, j}\right)$, the error term in (6.1) must be $\asymp T^{1 / 8} D^{-3 / 4}$ because the respective main terms in (6.2) cancel out. Moreover, since

$$
t_{2, j}-t_{1, j} \asymp D^{-1 / 2} T^{1 / 4}
$$

and

$$
\left|\frac{F\left(t_{2, j}\right)-F\left(t_{1, j}\right)}{t_{2, j}-t_{1, j}}\right| \asymp D^{-1 / 4} T^{-1 / 8},
$$

the bias of $Z(t)$ to the positive or negative direction for $t \in\left[t_{1, j}, t_{2, j}\right]$ is about $D^{-1 / 4} T^{-1 / 8}$.

\section{References}

[BoIw86] E. Bombieri, H. Iwaniec, On the order of $\zeta(1 / 2+i t)$, Ann. Scuola Norm. Sup. Pisa Cl. Sci. (4) 13 (1986), 449-472.

[Iv13] A. Ivić, The Theory of Hardy's Z - Function, Cambridge University Press, Cambridge, 2013.

[Jut09] M. Jutila, Atkinson's formula for Hardy's function, J. Number Theory 129 (2009), 2853-2878.

[Jut11] M. Jutila, An asymptotic formula for the primitive of Hardy's function, Arkiv Mat. 49 (2011), 97-107.

[Jut15] M. Jutila, The mean value of Hardy's function in short intervals, Indag. Mat. 26 (2015), 867-882.

[Jut17] M. Jutila, An approximate functional equation for the primitive of Hardy's function, Proc. Steklov Inst. Math. 299 (2017), 109-116.

[Kor08] M. A. Korolev, On the integral of Hardy's function $Z(t)$ (in Russian), Izv. Ross. Akad. Nauk, Ser. Mat. 72 (2008), 429-478.

[Leb72] N. N. Lebedev, Special functions and their applications, Dover, New York, 1972.

[Ti86] E. C. Titchmarsh, The Theory of the Riemann Zeta-function (2nd ed., revised by D. R. Heath-Brown), Clarendon Press, Oxford, 1986.

[Wat66] G. N. Watson, A Treatise on the Theory of Bessel functions, Cambridge University Press, 2nd ed., Cambridge, 1966.

\section{Matti Jutila}

Department of Mathematics and Statistics

University of Turku

FI-20014 Turku, Finland

e-mail: jutila@utu.fi 